\title{
L'urgence, élément constitutif des médias d'information de proximité sur Internet
}

\section{Sabine Brandicourt-Bertaux}

\section{(2) OpenEdition}

12 Journals

Édition électronique

URL : http://journals.openedition.org/communicationorganisation/3376

DOI : 10.4000/communicationorganisation.3376

ISSN : 1775-3546

Éditeur

Presses universitaires de Bordeaux

\section{Édition imprimée}

Date de publication : 1 juin 2006

Pagination : 85-92

ISSN : 1168-5549

\section{Référence électronique}

Sabine Brandicourt-Bertaux, «L'urgence, élément constitutif des médias d'information de proximité sur Internet », Communication et organisation [En ligne], 29 | 2006, mis en ligne le 19 juin 2012, consulté le 21 décembre 2020. URL : http://journals.openedition.org/communicationorganisation/3376 ; DOI : https://doi.org/10.4000/communicationorganisation.3376

Ce document a été généré automatiquement le 21 décembre 2020.

(c) Presses universitaires de Bordeaux 


\title{
L'urgence, élément constitutif des médias d'information de proximité sur Internet
}

\author{
Sabine Brandicourt-Bertaux
}

\section{Comment l'urgence influence les usages médiatiques de l'information de proximité}

1 Cette communication s'intéresse aux figures de l'urgence propres aux usages médiatiques de l'information de proximité. Cet article s'inscrit dans une recherche qui étudie les incidences de la mise en ligne sur le Web d'un genre informationnel spécifique, celui de l'information de proximité. Internet se caractérise comme un média qui défie les limites spatiales et temporelles. L'urgence, à l'instar de la territorialité, y prend donc une dimension spécifique.

2 La recherche d'une définition du paradigme « information de proximité » faisant partie de notre thèse, par convention pour cet article, l'expression «information de proximité » sera considérée comme synonyme $d^{\prime}$ ' information locale», de «microlocale ». La notion d' « information » est utilisée dans son sens journalistique. La notion de "proximité », qui contient en elle les aspects spatial, temporel, socioculturel, affectif, sera vue plutôt sous l'angle géographique. Les concepts de "médias de proximité » ou " presse de proximité » désignent ici la presse d'information régionale et locale, imprimée ou audio visuelle : la presse quotidienne régionale $(\mathrm{PQR})$, la presse hebdomadaire régionale (PHR), les journaux départementaux, cantonaux; les télévisions régionales, chaînes câblées et réseaux hertziens, télévisions de proximité, qu'elles soient du service public, privées, institutionnelles ou associatives; les radios locales publiques, privées ou associatives; les bulletins et supports de communication des institutions et collectivités locales... 


\section{L'urgence au quotidien}

3 Aujourd'hui, l'urgence est devenue la nouvelle mesure du temps social, ainsi que le démontre Zaki Laïdi, dans La Tyrannie de l'urgence : «[...] l'urgence ne constitue plus une catégorie temporelle extraordinaire et exceptionnelle. Elle s'impose comme la temporalité ordinaire de l'action en général. » (Laïdi, 1999, p. 30) De son côté, Nicole Aubert, dans Le Culte de l'urgence, parle du règne du temps court par rapport au temps long. (Aubert, 2003) Un domaine illustre particulièrement ce phénomène où l'urgence constitue la temporalité ordinaire: celui des médias. La préoccupation de l'immédiateté, du rapport au temps le plus court entre l'événement et l'annonce au public de cet événement, constitue un des moteurs de la presse, qu'elle soit écrite ou audiovisuelle. Tendre à l'information en temps réel est l'une des préoccupations des médias d'information. Pour répondre au public qui veut tout tout de suite, et donc dans l'urgence, la presse suit les évolutions technologiques qui défient l'espace et le temps et permettent de diversifier les moyens de communication. Les médias traditionnels (presse imprimée, radios, télévisions...) multiplient leurs offres pour proposer au public les informations qu'il attend. Ceci explique la mise en ligne du journal papier et la création, par les télévisions et radios, de sites Internet. Ainsi, par le multimédia, la presse écrite peut proposer de l'image animée et du son, la radio de l'écrit et de l'image, la télévision du texte en complément de ses images et du son. La mise en ligne raccourcit les délais de diffusion de l'information et rend le public moins tributaire des horaires et des zones de parution des éditions imprimées, ou de diffusion des émissions audiovisuelles.

Les médias de proximité, à l'instar de la presse nationale, ou même parfois en pionniers, se sont toujours intéressés de près aux possibilités des technologies de l'information et de la communication pour conquérir de nouveaux publics au sein de leur zone de diffusion mais aussi à l'extérieur. Lors du lancement du Minitel, plusieurs titres de la presse quotidienne régionale ont ouvert leur serveur pour diffuser des informations-services comme la météo, les résultats sportifs, scolaires, électoraux... La $\mathrm{PQR}$ s'est aussi investie dans les radios locales et les stations régionales de chaines télévisées pour diversifier ses supports. Aujourd'hui, certaines entreprises de presse mènent des expériences comme de transmettre des flashes d'informations sur les téléphones mobiles ou par courriel sur les messageries électroniques d'abonnés à ces services.

Quand on parle de proximité, un autre paramètre entre en ligne de compte, l'émotion, elle même mobilisée au sein de l'urgence comme l'explique Zaki Laïdi : «L'urgence mobilise une ressource décisive : l'émotion. [...] Or, par sa nature même, l'émotion a des conséquences temporelles incommensurables: elle disqualifie par avance toute contestation de cette immédiateté.» (Laïdi, 2000, p. 237) Zaki Laïdi évoque l'émotion relayée par les médias, à propos des aides humanitaires. Si l'on prend l'exemple de la catastrophe du tsunami en Asie en décembre 2004, l'émotion a effectivement eu un rôle important dans la mobilisation des peuples pour financer l'aide d'urgence. Les médias de proximité ajoutent à l'émotion les lois de la proxémique (proximité géographique, temporelle, socioculturelle, affective) : on s'intéresse d'abord à ce qui est proche de soi de par le lieu, le moment de l'événement, le lien social, la sphère privée (les «proches »)... 
6 Cependant, même si l'urgence s'est banalisée, sa gestion exige, de la part de la presse, une vigilance accrue, notamment au niveau de la vérification et de l'actualisation des informations. Dans le cas de l'information de proximité, cette exigence prend encore plus d'importance du fait justement de la proximité entre le média et son public. Les informations qu'annonce la presse de proximité ont des répercussions immédiates sur le terrain, en particulier en situation d'urgence. La vérification des informations ainsi que leur actualisation prend toute son importance dans cette situation. Une annonce produit des effets directement et presque dans l'immédiat par un effet de réactivité plus fort que lorsqu'il y a distance entre l'émetteur et le récepteur. La répercussion en chaîne des effets d'une annonce est encore accrue par le média Internet du fait de l'hypertextualité, l'instantanéité, la simultanéité et l'internationalisation.

\section{Temporalités médiatiques et proximité}

7 L'information de proximité implique une idée de l'urgence du quotidien, de type informations pratiques ou informations-services (météo, trafic routier, conseils de sécurité, services de garde...) La temporalité n'étant pas la même dans les différents médias, notamment en raison de la périodicité de parution ou du rythme des émissions, cette urgence du quotidien est traitée diversement.

Du fait des délais d'impression et de diffusion, ainsi que de la périodicité de publication, l'urgence dans la presse imprimée se trouve d'abord sous la forme préventive, puis sous la forme réactive, par le commentaire, avec des questions du genre « comment éviter cela?». Prenons un exemple : les services météorologiques prévoient des orages avec fortes pluies sur une zone donnée. Le journal pourra annoncer ces données. Mais au moment où les inondations se produisent, le quotidien ne pourra prévenir ses lecteurs $\mathrm{du}$ fait que la voie rapide est impraticable entre telle ou telle commune... Ce n'est que le lendemain que l'information pourra être publiée avec photos, analyses et commentaires (les causes des inondations, le moyen de les éviter à l'avenir, etc.) De ce fait, dans la presse quotidienne régionale, le mot «urgence» renvoie à la rubrique d'informations-services qui joue un rôle préventif: les "services de garde», par exemple, indiquent les coordonnées des médecins, pharmaciens, infirmiers de permanence ainsi que les numéros de téléphones des services d'urgence (policesecours, pompiers, SMUR, etc.) En revanche, une édition en ligne du quotidien pourrait afficher régulièrement l'état des routes par des flashes d'information.

9 La radio est davantage un média de l'immédiateté. Dans le cas des radios locales, ce sont les informations de sécurité routière et la météo qui relèvent de l'urgence. Par exemple, sur la fréquence 107.7, radio du service public qui diffuse sur le circuit autoroutier français, une information concernant un obstacle au point kilomètre $n$ est important pour les automobilistes roulant à proximité et à cet instant précis. La situation d"urgence ne durera que le temps où cet obstacle sera sur l'autoroute. Ce type d'information est diffusé au moment des "points infos": point circulation, point météo... Cependant, en raison du degré d'urgence, l'information peut être donnée immédiatement à l'antenne, interrompant l'émission en cours. Du point de vue de la rapidité de diffusion de l'information, la radio est aussi performante qu'Internet et même davantage si l'on tient compte des délais de connexion au Web.

10 A la télévision, tributaire des horaires de diffusion, ce flash d'urgence pourra apparaître sous forme d'un sous-titrage ou d'une intervention en direct d'un 
journaliste au milieu d'une émission. Dans le cas d'une information de proximité urgente, les décrochages régionaux des antennes nationales sont peu souples en dehors des horaires de diffusion habituelle. C'est là où le site Internet de l'antenne régionale en question aura un rôle à jouer, le Web n'étant pas tributaire des horaires de diffusion. S'affichera alors sur l'écran de télévision une mention du genre : « l'info en continu sur www.france3.fr » qui renverra au réseau.

11 Créer un site Internet, pour un média de proximité, est la possibilité de passer outre les contraintes de périodicité, de publication ou d'émission, et de zones de diffusions. Cependant, même par ce biais, l'immédiateté reste illusoire car il existe toujours un décalage entre le temps de l'actualité, le temps de l'écriture et le temps de lecture (temporalités analysées par Anne-Marie Jannet et Claude Jamet, dans l'article « Le jeu du présent » in Médias, temporalités et démocratie, 2000).

Luc Bonneville, qui étudie la temporalité du réseau Internet (« La temporalité du réseau Internet est-elle encore moderne? », in: Internet, nouvel espace citoyen, 2002), note que les usagers d'Internet à domicile construisent leur «temps présent » par rapport à un " temps à soi ", non seulement en menant plusieurs tâches en même temps mais aussi au moment souhaité tant pour consulter des informations que pour en envoyer sur le réseau. De ce fait, l'instantanéité devient plus individuelle que sociale. L'omniprésence de l'urgence comme mode d'action glisse du monde professionnel à l'espace privé, où la demande d'information de proximité est forte. Par conséquent, l'information de proximité n'échappe pas à la règle de l'urgence.

\section{Le réseau d'informateurs de proximité dans l'organisation communicationnelle}

13 La presse recueille ses informations par le biais de ses journalistes, et, dans le cas de la presse de proximité, par ses correspondants locaux. Lorsqu'un événement imprévu survient, la qualité des informations est proportionnelle à la qualité du réseau d'informateurs et à leur réactivité. Dans le cas du traitement de l'information au jour le jour, le journaliste tient compte de ces informations reçues, les vérifie avant de les publier. Si le temps de diffusion se raccourcit, notamment en cas d'urgence, il faut qu'il est les moyens de s'assurer une rapidité de vérification de l'information.

Les NTIC ont permis d'améliorer l'acheminement des informations de la source jusqu'à la publication en passant par la rédaction qui traite l'information : télécopie, courriel, transmission d'images numérisées, PAO, téléphonie mobile, Internet... L'utilisation des NTIC n'est pas réservée aux professionnels. On voit ainsi que n'importe quel témoin d'un événement, muni d'un téléphone portable, devient relais de l'information, d'autant plus si ce téléphone fait office d'appareil photo ou si ce témoin est muni d'une caméra ou d'une «webcam». Chacun peut s'improviser informateur et diffuser immédiatement au monde entier les images et le son de l'événement auquel il vient d'assister. On voit le rôle que peuvent jouer, en situation d'urgence, ces informateurs improvisés.

15 Les NTIC ont aussi facilité le développement à partir des années 1980-1990 des services de communication dans les organisations. Ainsi, les collectivités locales se sont dotées de supports où l'information de proximité est déclinée dans son aspect citoyenneté. Les organisations se sont alors préoccupées d'améliorer leurs relations avec la presse, 
notamment pour mieux maîtriser les situations de crise. Des procédures d'urgence ont été mises en place en communication organisationnelle. La gestion de l'urgence devient tributaire d'une bonne organisation communicationnelle, aussi bien en interne qu'en externe, s'appuyant sur un maillage d'informateurs. Les institutions ont donc créé leurs sites Internet en espérant pouvoir répondre aux attentes du public. Une bonne gestion de l'urgence demande, comme pour toute situation de crise, d'anticiper, de suivre l'évolution de la crise et d'en garder la traçabilité. La mise à jour des informations sur le site Internet institutionnel doit s'approcher le plus possible du «temps réel» car le public internaute attend que cet outil lui donne cette information réactualisée en permanence. Roselyne Ringoot parle même, à ce propos, d'information perpétuelle ( $R$. Ringoot, "L'information perpétuelle. Les constructions temporelles dans l'Internet local»in Inform@tion.local, 2002, p. 293-305). Elle souligne que les flashes d'information réactualisés régulièrement, une fois périmés, viennent constituer les archives, mais ces archives sont disponibles simultanément sur le site. Ce qui l'amène à cette conclusion, «[...] le potentiel de stockage des contenus et le potentiel de temps réel provoquent une dilution des périodicités... l'Internet immobilise le temps plus qu'il ne l'accélère. » Par rapport au traitement de l'urgence par les médias de proximité, cette réflexion apporte une perspective: Internet peut constituer un outil de la traçabilité de la gestion de l'urgence...

16 Le défi de la réactualisation permanente n'est pas simple à relever, non seulement pour les collectivités locales, mais aussi pour tous les producteurs d'information de proximité. La stratégie, pour améliorer la gestion de l'urgence, est alors de densifier le réseau des informateurs et d'utiliser toutes les ressources d'Internet comme l'interactivité, le multimédia, la couverture mondiale. Un des moyens de s'en sortir est d'utiliser les possibilités de l'hypertexte de la Toile mondiale, en renvoyant vers les sites spécialisés ou en diffusant les informations directement puisées à la source sans retraitement. Ainsi, le site de La Voix du Nord en ligne propose une rubrique météo qui renvoie à « Météo France » et le point sur la circulation propose des liens vers des sites spécialisés (Equipement, Centre régional d'information sur la sécurité routière, services de plans et d'itinéraires en ligne, etc.)

17 Cependant, une analyse des sites Internet des médias de proximité français montre que chaque média traditionnel reste proche de son savoir faire initial, de son métier d'origine et n'exploite pas toutes les possibilités des NTIC pour apporter un meilleur service à son public. Ainsi, si pour les informations nationales, les dépêches AFP sont publiées sur l'édition en ligne du quotidien régional au fur et à mesure de leur diffusion, en revanche, les informations locales du jour, même diffusées par l'AFP, ne sont publiées que le lendemain, en même temps que sur les éditions imprimées. Pourtant, en situation d'urgence, ces médias pourraient mettre en ligne, au fur et à mesure de leur arrivée à la rédaction, les informations recueillies sur le terrain par leurs journalistes et correspondants locaux. Peut-être faut-il attendre des événements exceptionnels? Ainsi, lors des attentats du 11 septembre 2001 à New-York (Etats-Unis), les journaux d'information générale du monde entier ont rapidement réagi sur leurs éditions en ligne, comme le souligne l'introduction de l'ouvrage Inform@tion.local (Damian, Ringoot, Thierry, Ruellan, 2002). Voilà des perspectives pour les médias de proximité qui souhaiteraient améliorer la couverture médiatique et le traitement de l'urgence de proximité... 


\section{BIBLIOGRAPHIE}

Aubert, N., Le culte de l'urgence, Paris, Flammarion, 2003.

Damian, B., Ringoot, R., Thierry, D., Ruellan, D. (sous la direction de), Inform@tion.local, Le paysage médiatique à l'ère électronique, Paris, L'Harmattan, 2002.

Jauréguiberry, F., Proulx, S. (sous la direction de), Internet, nouvel espace citoyen, Paris, L'Harmattan, 2002.

Laïdi, Z., Le sacre du présent, Paris, Flammarion, 2000.

Laïdi, Z., La tyrannie de l'urgence, Montréal, Fides, 1999.

Vitalis, A., Tétu, J.-F., Palmer, M., Castagna, B. (sous la direction de), Médias, temporalités et démocratie, Rennes, Apogée/PUF, 2000.

\section{RÉSUMÉS}

Les usages médiatiques de l'information de proximité se transforment avec les nouvelles technologies de l'information et de la communication (NTIC) et notamment Internet. Cet article propose de démontrer comment l'urgence devient l'un des éléments centraux de ces nouveaux usages. Pour répondre au public qui veut tout tout de suite, et donc dans l'urgence, la presse de proximité suit les évolutions technologiques qui défient l'espace et le temps et permettent de diversifier les moyens de communication.

The media uses of the information of proximity change with new communication and information technologies (NCITs) and in particular Internet. This article proposes to show how the urgency becomes one of the central elements of these new uses. To answer the public which wants all immediately, and thus in the urgency, the press of proximity follows the technological developments who defy space and time and allow to diversify the means of communication.

\section{INDEX}

Mots-clés : NTIC, médias, presse de proximité

\section{AUTEUR}

\section{SABINE BRANDICOURT-BERTAUX}

Sabine Brandicourt-Bertaux est doctorante à l'université Charles-de-Gaulle à Lille 3, à l'UMR CERSATES, équipe GERIICO. Son domaine de recherche est l'information de proximité ainsi que l'évolution des usages médiatiques. Mail :

sabine.bertaux@laposte.net 